\title{
On critical points of Gaussian random fields under diffeomorphic transformations
}

\author{
Dan Cheng* \\ Arizona State University \\ Armin Schwartzman ${ }^{\dagger}$
University of California, San Diego
}

November 20, 2019

\begin{abstract}
Let $\{X(t), t \in M\}$ and $\left\{Z\left(t^{\prime}\right), t^{\prime} \in M^{\prime}\right\}$ be smooth Gaussian random fields parameterized on Riemannian manifolds $M$ and $M^{\prime}$, respectively, such that $X(t)=Z(f(t))$, where $f: M \rightarrow M^{\prime}$ is a diffeomorphic transformation. We study the expected number and height distribution of the critical points of $X$ in connection with those of $Z$. As an important case, when $X$ is an anisotropic Gaussian random field, then we show that its expected number of critical points becomes proportional to that of an isotropic field $Z$, while the height distribution remains the same as that of $Z$.
\end{abstract}

Keywords: Gaussian random fields; Diffeomorphic transformation; Critical points; Expected number; Height distribution; Anisotropic; Isotropic.

Mathematics Subject Classification: 60G15, 60G60, 15B52.

\section{Introduction}

The study of critical points of Gaussian random fields, especially of their expected number and height distribution, is important in probability theory [1, 8] and has applications in many areas such as physics [10], statistics [7, 4], neuroimaging [14, 13] and astronomy [3]. However, the exact formulae for the expected number and height distribution of critical points of Gaussian fields are difficult of obtain. The only exception, so far to the authors' knowledge, is the isotropic Gaussian fields [5, 6]. In this paper, we investigate Gaussian fields under diffeomorphic transformations and find a new approach to obtain the exact formulae for a wider class of Gaussian fields.

An important case is the anisotropic Gaussian field, which is a useful model in spatial statistics [2]. Anisotropic fields also appear in smoothing of 3D brain images. While isotropic

\footnotetext{
${ }^{*}$ Research partially supported by NSF grant DMS-1902432.

${ }^{\dagger}$ Research partially supported by NSF grant DMS-1811659.
} 
kernels are often used, it has been shown that in some situations anisotropic kernels yield better results [15].

The standard approach for solving the expected number and height distribution of critical points of Gaussian random fields is the Kac-Rice formula [1, 10. However, the problem becomes much simpler by realizing that an anisotropic field can be obtained by a linear transformation of the index parameter of an isotropic field; see Section 3.1 below. We present here an easy solution to the expected number and height distribution of critical points of anisotropic Gaussian fields by casting the problem more generally as a diffeomorphic transformation of the parameter space.

This approach has advantages for other applications where diffeomorphic transformations are used. Diffeomorphic transformations of random fields appear, for example, in structural and functional imaging of the cortical surface of the brain. Because the brain's cortex is a convoluted closed surface, it is often mapped onto the 2-dimensional sphere $\mathbb{S}^{2}[9]$, where statistical inference can be performed using Gaussian random field theory [12, 13]. Thus, the study of topological features of quantities such as local maxima and local minima of cortical thickness and cortical activity can be carried out in a simpler representation on the sphere.

\section{Gaussian Random Fields under Diffeomorphic Transforma- tions}

Let $\{X(t), t \in M\}$ be a smooth Gaussian random field parameterized on a piecewise $C^{2}$ compact Riemannian manifold $M$. Here and in the sequel, the smoothness assumption means that the field satisfies the condition (11.3.1) in [1], which is slightly stronger than $C^{2}$ but can be implied by $C^{3}$. The number of critical points of index $i$ of $X$ exceeding level $u$ over the domain $M$ is defined as

$$
\mu_{i}^{X}(M, u)=\#\left\{t \in M: X(t) \geq u, \nabla X(t)=0, \operatorname{index}\left(\nabla^{2} X(t)\right)=i\right\}, \quad 0 \leq i \leq N,
$$

where $\nabla X(t)$ and $\nabla^{2} X(t)$ are respectively the gradient and Hessian of $X$, and index $\left(\nabla^{2} X(t)\right)$ denotes the number of negative eigenvalues of $\nabla^{2} X(t)$. The expected number of critical points is therefore $\mathbb{E}\left[\mu_{i}^{X}(M, u)\right]$.

We introduce next the height distribution of a critical point of $X$. It is convenient to consider the tail probability, that is, the probability that the height of the critical point exceeds a fixed threshold at that point, conditioned on the event that the point is a critical point of $X$. Such conditional probability can be defined as

$$
F_{i}^{X}(t, u)=\mathbb{P}\{X(t)>u \mid t \text { is a critical point of index } i \text { of } X\} .
$$

It is shown in [5] that the height distribution of a critical point of index $i$ of $X$ at $t$ is

$$
F_{i}^{X}(t, u)=\frac{\mathbb{E}\left\{\left|\operatorname{det}\left(\nabla^{2} X(t)\right)\right| \mathbb{1}_{\{X(t)>u\}} \mathbb{1}_{\left\{\operatorname{index}\left(\nabla^{2} X(t)\right)=i\right\}} \mid \nabla X(t)=0\right\}}{\mathbb{E}\left\{\left|\operatorname{det}\left(\nabla^{2} X(t)\right)\right| \mathbb{1}_{\left\{\operatorname{index}\left(\nabla^{2} X(t)\right)=i\right\}} \mid \nabla X(t)=0\right\}} .
$$


A mapping $f: M \rightarrow M^{\prime}$ is called $C^{2}$ diffeomorphic if it is one-to-one and $f$ and its inverse $f^{-1}$ are both twice differentiable. Suppose there exists $\left\{Z\left(t^{\prime}\right), t^{\prime} \in M^{\prime}\right\}$, a smooth Gaussian random field parameterized on a Riemannian manifold $M^{\prime}$, and a $C^{2}$ diffeomorphic transformation $f: M \rightarrow M^{\prime}$, such that $X(t)=Z(f(t))$. Similarly to (2.1), the number of critical points of index $i$ of $Z$ exceeding level $u$ over the domain $M^{\prime}$ is defined as

$$
\mu_{i}^{Z}\left(M^{\prime}, u\right)=\#\left\{t^{\prime} \in M^{\prime}: Z\left(t^{\prime}\right) \geq u, \nabla Z\left(t^{\prime}\right)=0, \operatorname{index}\left(\nabla^{2} Z\left(t^{\prime}\right)\right)=i\right\}, \quad 0 \leq i \leq N
$$

and the height distribution of critical points of index $i$ of $Z$ at $t^{\prime}$ is defined as $F_{i}^{Z}\left(t^{\prime}, u\right)$.

We have the following main result.

Theorem 2.1 Let $\{X(t), t \in M\}$ and $\left\{Z\left(t^{\prime}\right), t^{\prime} \in M^{\prime}\right\}$ be smooth Gaussian random fields parameterized on $N$-dimensional, piecewise $C^{2}$, compact Riemannian manifolds $M$ and $M^{\prime}$, respectively, such that $X(t)=Z(f(t))$, where $f: M \rightarrow M^{\prime}$ is a $C^{2}$ diffeomorphic transformation. Then for $0 \leq i \leq N, t \in M$ is a critical point of index $i$ of $X$ if and only if $t^{\prime}=f(t) \in M^{\prime}$ is a critical point of index $i$ of $Z$; and moreover, for $u \in \mathbb{R}$,

$$
\mathbb{E}\left[\mu_{i}^{X}(M, u)\right]=\mathbb{E}\left[\mu_{i}^{Z}\left(M^{\prime}, u\right)\right] \quad \text { and } \quad F_{i}^{X}(t, u)=F_{i}^{Z}\left(t^{\prime}, u\right) .
$$

Proof. Denote by $T_{t} M$ and $T_{t^{\prime}} M^{\prime}$ the tangent spaces at $t \in M$ and $t^{\prime} \in M^{\prime}$, respectively. By the chain rule,

$$
\left.d X\right|_{t}=\left.\left.d Z\right|_{f(t)} d f\right|_{t}
$$

where $\left.d X\right|_{t}: T_{t} M \rightarrow \mathbb{R},\left.d f\right|_{t}: T_{t} M \rightarrow T_{f(t)} M^{\prime}$ and $\left.d Z\right|_{f(t)}: T_{f(t)} M^{\prime} \rightarrow \mathbb{R}$. We may write

$$
\begin{aligned}
\left.d X\right|_{t} & =\left.\sum_{i=1}^{N} \frac{\partial X}{\partial x_{i}} d x_{i}\right|_{t}, \\
\left.d Z\right|_{f(t)} & =\left.\sum_{i=1}^{N} \frac{\partial Z}{\partial x_{i}^{\prime}} d x_{i}^{\prime}\right|_{f(t)},
\end{aligned}
$$

and $\left.d f\right|_{t}$ is a linear mapping that can be represented as an $N \times N$ matrix, denoted by $B=$ $\left(B_{i j}\right)_{1 \leq i, j \leq N}$, such that

$$
\left.d f\right|_{t}(v)=\left.\sum_{i=1}^{N}\left(\sum_{j=1}^{N} B_{i j} v_{j}\right) \frac{\partial}{\partial x_{i}^{\prime}}\right|_{f(t)}, \quad \forall v=\left.\sum_{i=1}^{N} v_{i} \frac{\partial}{\partial x_{i}}\right|_{t} \in T_{t} M,
$$

where $\left\{\left.\frac{\partial}{\partial x_{i}}\right|_{t}\right\}_{i=1}^{n}$ and $\left\{\left.\frac{\partial}{\partial x_{i}^{\prime}}\right|_{f(t)}\right\}_{i=1}^{n}$ are the bases in $T_{t} M$ and $T_{f(t)} M^{\prime}$, respectively. Let $\left.e_{i}\right|_{t}=$ $\left.\frac{\partial}{\partial x_{i}}\right|_{t}$. By (2.4) and (2.5), we have

$$
\begin{aligned}
\left.\frac{\partial X}{\partial x_{i}}\right|_{t} & =\left\langle\left. d X\right|_{t},\left.\frac{\partial}{\partial x_{i}}\right|_{t}\right\rangle=\left.d X\right|_{t}\left(\left.\frac{\partial}{\partial x_{i}}\right|_{t}\right)=\left.d X\right|_{t}\left(\left.e_{i}\right|_{t}\right) \\
& =\left.\left.d Z\right|_{f(t)} d f\right|_{t}\left(\left.e_{i}\right|_{t}\right)=\left.d Z\right|_{f(t)}\left(\left.\sum_{k=1}^{N} B_{k i} \frac{\partial}{\partial x_{k}^{\prime}}\right|_{f(t)}\right)=\left.\sum_{k=1}^{N} B_{k i} \frac{\partial Z}{\partial x_{k}^{\prime}}\right|_{f(t)} .
\end{aligned}
$$


Therefore,

$$
\left.\nabla X\right|_{t}=\left(\frac{\partial X}{\partial x_{1}}, \ldots, \frac{\partial X}{\partial x_{N}}\right)^{T}=B^{T}\left(\frac{\partial Z}{\partial x_{1}^{\prime}}, \ldots, \frac{\partial Z}{\partial x_{N}^{\prime}}\right)^{T}=\left.B^{T} \nabla Z\right|_{f(t)}
$$

implying

$$
\left.\nabla X\right|_{t}=\left.0 \quad \Leftrightarrow \quad \nabla Z\right|_{f(t)}=0
$$

Similarly, we have

$$
\left.\frac{\partial}{\partial x_{j}}\left(\frac{\partial X}{\partial x_{i}}\right)\right|_{t}=\left.\sum_{l=1}^{N} \sum_{k=1}^{N} B_{k i} \frac{\partial}{\partial x_{l}^{\prime}}\left(\frac{\partial Z}{\partial x_{k}^{\prime}}\right)\right|_{f(t)} B_{l j} .
$$

Therefore

$$
\left.\nabla^{2} X\right|_{t}=\left.B^{T} \nabla^{2} Z\right|_{f(t)} B
$$

implying

$$
\operatorname{index}\left(\left.\nabla^{2} X\right|_{t}\right)=\operatorname{index}\left(\left.\nabla^{2} Z\right|_{f(t)}\right) .
$$

It follows from (2.6) and (2.8) that $t \in M$ is a critical point of index $i$ of $X$ if and only if $t^{\prime}=f(t) \in M^{\prime}$ is a critical point of index $i$ of $Z$.

It follows from (2.1), (2.6) and (2.8) that

$$
\begin{aligned}
\mu_{i}^{X}(M, u) & =\#\left\{t \in M: X(t) \geq u, \nabla X(t)=0, \operatorname{index}\left(\nabla^{2} X(t)\right)=i\right\} \\
& =\#\left\{t \in M: Z(f(t)) \geq u,\left.\nabla Z\right|_{f(t)}=0, \operatorname{index}\left(\left.\nabla^{2} Z\right|_{f(t)}\right)=i\right\} \\
& =\mu_{i}^{Z}\left(M^{\prime}, u\right),
\end{aligned}
$$

yielding $\mathbb{E}\left[\mu_{i}^{X}(M, u)\right]=\mathbb{E}\left[\mu_{i}^{Z}\left(M^{\prime}, u\right)\right]$.

Notice that (2.7) implies $\operatorname{det}\left(\nabla^{2} X(t)\right)=|\operatorname{det}(B)|^{2} \operatorname{det}\left(\left.\nabla^{2} Z\right|_{f(t)}\right)$. Therefore, by (2.2), (2.6) and (2.8),

$$
\begin{aligned}
F_{i}^{X}(t, u) & =\frac{\mathbb{E}\left\{\left|\operatorname{det}\left(\nabla^{2} X(t)\right)\right| \mathbb{1}_{\{X(t)>u\}} \mathbb{1}_{\left\{\operatorname{index}\left(\nabla^{2} X(t)\right)=i\right\}} \mid \nabla X(t)=0\right\}}{\mathbb{E}\left\{\left|\operatorname{det}\left(\nabla^{2} X(t)\right)\right| \mathbb{1}_{\left\{\operatorname{index}\left(\nabla^{2} X(t)\right)=i\right\}} \mid \nabla X(t)=0\right\}} \\
& =\frac{|\operatorname{det}(B)|^{2} \mathbb{E}\left\{\left|\operatorname{det}\left(\left.\nabla^{2} Z\right|_{f(t)}\right)\right| \mathbb{1}_{\{Z(f(t))>u\}} \mathbb{1}_{\left\{\operatorname{index}\left(\left.\nabla^{2} Z\right|_{f(t)}\right)=i\right\}}|\nabla Z|_{f(t)}=0\right\}}{|\operatorname{det}(B)|^{2} \mathbb{E}\left\{\left.\left|\operatorname{det}\left(\left.\nabla^{2} Z\right|_{f(t)}\right)\right|\left|\mathbb{1}_{\left\{\operatorname{index}\left(\left.\nabla^{2} Z\right|_{f(t)}\right)=i\right\}}\right| \nabla Z\right|_{f(t)}=0\right\}} \\
& =F_{i}^{Z}\left(t^{\prime}, u\right) .
\end{aligned}
$$

Remark 2.2 In certain situations, we only know the covariance, or equivalently the distribution, of $X(t)$. But there exist another Gaussian field $Z$ and some diffeomorphic transformation $f$ such that $X(t)$ and $Z(f(t))$ have the same distribution, that is $X(t) \stackrel{d}{=} Z(f(t))$. Then the results in (2.3) still hold since the expected number and height distribution of critical points depend only on the distribution of the field. 


\section{Applications}

\subsection{Anisotropic Gaussian Random Fields on Euclidean Space}

Let $A$ be a nondegenerate $N \times N$ matrix, that is $\operatorname{det}(A) \neq 0$. An anisotropic Gaussian random field $\left\{X(t), t \in \mathbb{R}^{N}\right\}$ is defined as

$$
X(t)=Z(A t), \quad t \in \mathbb{R}^{N},
$$

where $\left\{Z(t), t \in \mathbb{R}^{N}\right\}$ is a smooth, unit-variance, isotropic Gaussian random field. So, it is a special case of the diffeomorphic transformation $f: \mathbb{R}^{N} \rightarrow \mathbb{R}^{N}$ where $f(t)=A t$. Due to the isotropy of $Z$, there exists a function $\rho:[0, \infty) \rightarrow \mathbb{R}$ such that the covariance of $Z$ has the form

$$
\mathbb{E}[Z(t) Z(s)]=\rho\left(\|t-s\|^{2}\right) .
$$

By (3.1) and (3.2), equivalently, we may call $\left\{X(t), t \in \mathbb{R}^{N}\right\}$ an anisotropic Gaussian random field if the covariance has the form

$$
\mathbb{E}[X(t) X(s)]=\rho\left(\|A(t-s)\|^{2}\right) .
$$

Using the isotropy property (3.2), it has been shown in Cheng and Schwartzman [5, 6] that the exact formulae of the expected number and height distribution of critical points of the isotropic Gaussian field $Z$ can be obtained by using GOI random matrices. We show below that the study of critical points of anisotropic Gaussian fields can be transferred to isotropic Gaussian fields, so that their expected number and height distribution can be obtained exactly.

Corollary 3.1 Let $\left\{X(t), t \in \mathbb{R}^{N}\right\}$ be a smooth, unit-variance, anisotropic Gaussian random field satisfying (3.1) and let $D$ be an $N$-dimensional Jordan measurable set on $\mathbb{R}^{N}$. Then for $0 \leq i \leq N$ and $u \in \mathbb{R}$,

$$
\mathbb{E}\left[\mu_{i}^{X}(D, u)\right]=|\operatorname{det}(A)| \mathbb{E}\left[\mu_{i}^{Z}(D, u)\right] \text { and } F_{i}^{X}(u)=F_{i}^{Z}(u),
$$

where $\mathbb{E}\left[\mu_{i}^{Z}(D, u)\right]$ and $F_{i}^{Z}(u)$ are respectively the expected number and height distribution for the isotropic Gaussian field $Z$ that can be found in [5, 6]; and $F_{i}^{X}(u):=F_{i}^{X}(t, u)$ and $F_{i}^{Z}(u):=$ $F_{i}^{Z}(t, u)$ since they do not depend on $t$ due to the stationarity (isotropy) of $X$ and $Z$.

Proof. Let $D^{\prime}=\{A t: t \in D\}$, then $\operatorname{Vol}\left(D^{\prime}\right)=|\operatorname{det}(A)| \operatorname{Vol}(D)$. It follows from Theorem 2.1 and the isotropy of $Z$ that

$$
\mathbb{E}\left[\mu_{i}^{X}(D, u)\right]=\mathbb{E}\left[\mu_{i}^{Z}\left(D^{\prime}, u\right)\right]=|\operatorname{det}(A)| \operatorname{Vol}(D) \mathbb{E}\left[\mu_{i}^{Z}\left([0,1]^{N}, u\right)\right]=|\operatorname{det}(A)| \mathbb{E}\left[\mu_{i}^{Z}(D, u)\right]
$$

and

$$
F_{i}^{X}(u)=F_{i}^{X}(t, u)=F_{i}^{Z}(A t, u)=F_{i}^{Z}(t, u)=F_{i}^{Z}(u) .
$$


Remark 3.2 Corollary 3.1 shows that the expected number of critical points of the anisotropic field $X$ is that of the isotropic field $Z$ scaled by $|\operatorname{det}(A)|$, while the height distribution remains the same as that of $Z$. A special case when $A$ is a diagonal matrix was considered in [11] with tedious computation.

It can also be seen easily that the results in Corollary 3.1 hold in a more general setting where $Z$ is stationary but not necessarily isotropic.

\subsection{Gaussian Random Fields on Ellipsoids}

Suppose $\{X(t), t \in M\}$ is a smooth Gaussian field on an $N$-dimensional ellipsoid $M$. If $M$ is embedded in $\mathbb{R}^{N+1}$, then there is a linear mapping on $\mathbb{R}^{N+1}$, denoted by $g$, that maps $M$ onto the $N$-dimensional unit sphere $\mathbb{S}^{N}$. Let $f: M \rightarrow \mathbb{S}^{N}$ be the $N$-dimensional diffeomorphic mapping between $M$ and $\mathbb{S}^{N}$ induced by $g$. Then we can write $X(t)=Z(f(t))$, where $\left\{Z\left(t^{\prime}\right), t^{\prime} \in \mathbb{S}^{N}\right\}$ is a Gaussian random field on the $N$-dimensional unit sphere $\mathbb{S}^{N}$. Now the study of critical points of $X$ on the ellipsoid $M$ can be transferred to the Gaussian field $Z$ on the unit sphere $\mathbb{S}^{N}$ by Theorem 2.1 .

In particular, if the Gaussian field $Z$ above is isotropic on $\mathbb{S}^{N}$, then by Theorem 2.1 ,

$\mathbb{E}\left[\mu_{i}^{X}(M, u)\right]=\mathbb{E}\left[\mu_{i}^{Z}\left(\mathbb{S}^{N}, u\right)\right]$ and $F_{i}^{X}(t, u)=F_{i}^{Z}\left(t^{\prime}, u\right)$ with $t^{\prime}=f(t)$, whose formulae can be found in [5, 6].

\section{Acknowledgment}

The first author thanks Professor Yan Fyodorov for helpful discussions.

\section{References}

[1] Adler, R. J. and Taylor, J. E. (2007). Random Fields and Geometry. Springer, New York.

[2] Allard, D., Senoussi, R. and Porcu, E. (2016) Anisotropy Models for Spatial Data. Mathematical Geosciences. 48, 305-328.

[3] Bardeen, J. M., Bond, J. R., Kaiser, N. and Szalay A. S. (1985). The statistics of peaks of Gaussian random fields. Astrophys. J., 304, 15-61.

[4] Cheng, D., Cammarota, V., Fantaye, Y., Marinucci, D. and Schwartzman, A. (2019). Multiple testing of local maxima for detection of peaks on the (celestial) sphere. Bernoulli, in press. arXiv:1602.08296

[5] Cheng, D. and Schwartzman, A. (2015). Distribution of the height of local maxima of Gaussian random fields. Extremes, 18, 213-240.

[6] Cheng, D. and Schwartzman, A. (2018). Expected number and height distribution of critical points of smooth isotropic Gaussian random fields. Bernoulli, 24, 3422-3446.

[7] Cheng, D. and Schwartzman, A. (2017). Multiple testing of local maxima for detection of peaks in random fields. Ann. Stat., 45, 529-556. 
[8] Cramér, H. and Leadbetter, M. R. (1967). Stationary and Related Stochastic Processes: Sample Function Properties and Their Applications. Wiley, New York.

[9] Fischl, B., Sereno, M. I., and Dale, A. M. (1999). Cortical surface-based analysis: II: inflation, flattening, and a surface-based coordinate system. Neuroimage, 9(2), 195-207.

[10] Fyodorov, Y. V. (2004). Complexity of random energy landscapes, glass transition, and absolute value of the spectral determinant of random matrices. Phys. Rev. Lett., 92, 240601.

[11] Fyodorov, Y. V. (2015). High-Dimensional Random Fields and Random Matrix Theory. Markov Processes and Related Fields, 21, 483-518.

[12] Hagler Jr, D. J., Saygin, A. P., and Sereno, M. I. (2006). Smoothing and cluster thresholding for cortical surface-based group analysis of fMRI data. Neuroimage, 33(4), 1093-1103.

[13] Pantazis, D., Nichols, T. E., Baillet, S., and Leahy, R. M. (2005). A comparison of random field theory and permutation methods for the statistical analysis of MEG data. Neuroimage, 25(2), 383-394.

[14] Taylor, J. E. and Worsley, K. J. (2007). Detecting sparse signals in random fields, with an application to brain mapping. J. Am. Statist. Assoc., 102, 913-928.

[15] Van Hecke, W., Leemans, A., De Backer, S., Jeurissen, B., Parizel, P. M., and Sijbers, J. (2010). Comparing isotropic and anisotropic smoothing for voxelbased DTI analyses: A simulation study. Human brain mapping, 31(1), 98-114.

\section{Dan Cheng}

School of Mathematical and Statistical Sciences

Arizona State University

900 S. Palm Walk

Tempe, AZ 85281, U.S.A.

E-mail: cheng.stats@gmail.com

ARmin SchWartzman

Division of Biostatistics and Bioinformatics and

Halicioğlu Data Science Institute

University of California San Diego

9500 Gilman Dr.

La Jolla, CA 92093, U.S.A.

E-mail: armins@ucsd.edu 